Jean-Christophe Feauveau, Institut Préparatoire aux Etudes Scientifiques et Techniques, Route de Sidi Bou Saï, 2070 La Marsa, Tunisia. e-mail:

jean-christophe.feauveau@supelec-rennes.fr

\title{
A GENERALIZED RIEMANN INTEGRAL FOR BANACH-VALUED FUNCTIONS
}

\begin{abstract}
We shall develop the properties of an integral for Banach-valued functions. The formalism is the generalized Riemann integral introduced by Kurzweil [5] and Henstock [4]. More precisely, the presentation is close to the McShane approach [6]. Besides its simplicity of presentation, four advantages characterize this theory:

(i) the definition can be used for real-valued functions, and can be generalized without modification to general real and complex Banach spaces;

(ii) when a function is integrable its norm is also integrable, and the proof is straightforward from the definition;

(iii) for finite dimension spaces the theory is equivalent to the McShane's theory, which is itself equivalent to the Lebesgue's theory;

(iv) and lastly, for general Banach space, we can prove the equivalence to the Bochner's theory.
\end{abstract}

\section{Gauges, Tagged Partitions of $[a, b]$ and Conventions}

In the following, $(X,\|\|)$ will denote a Banach space.

Let $[a, b]$ be a real interval, $(a<b)$. By definition, a gauge on $[a, b]$ is a function $\delta$ from $[a, b]$ to $\mathbb{R}_{+}^{*}$.

Following the McShane definition [6], a tagged partition $\left(\left(\left[x_{i-1}, x_{i}\right]\right)_{1 \leq i \leq n}\right.$, $\left.\left(c_{i}\right)_{1 \leq i \leq n}\right)$, is a couple of finite sequences where the closed intervals $\left(\left[x_{i-1}, x_{i}\right]\right)_{1 \leq i \leq n}$ form a partition of $[a, b]$ and the numbers $\left(c_{i}\right)_{1 \leq i \leq n}$ are called the corresponding tags. $\delta$ if

A tagged partition $\left(\left(\left[x_{i-1}, x_{i}\right]\right)_{1 \leq i \leq n},\left(c_{i}\right)_{1 \leq i \leq n}\right)$ is subordinate to a gauge

$$
\forall i \in\{1, \ldots, n\}, \quad c_{i}-\delta\left(c_{i}\right) \leq x_{i-1}<x_{i} \leq c_{i}+\delta\left(c_{i}\right) .
$$

Key Words: McShane integral, Bochner integral, vector-valued integral

Mathematical Reviews subject classification: 26A39, 28B05

Received by the editors October 15, 1999 
In order to simplify we write $(x, c)$, where $x=\left(\left[x_{i-1}, x_{i}\right]\right)_{1 \leq i \leq n}$ and $c=$ $\left(c_{i}\right)_{1 \leq i \leq n}$.

And lastly, for a tagged partition $(x, c)$ of $[a, b]$, the Riemann sum of $f$ : $[a, b] \rightarrow X$ for this partition is

$$
S_{f}(x, c)=\sum_{i}\left(x_{i}-x_{i-1}\right) f\left(c_{i}\right) .
$$

\section{Definitions and Characterization}

Throughout the paper, a function $f:[a, b] \rightarrow E$ is called integrable if, for each $\varepsilon>0$, we can find a gauge $\delta_{\varepsilon}$ such that

$$
\sum_{i}\left\|\left(x_{i}-x_{i-1}\right)\left(f\left(c_{i}\right)-f\left(c_{i}^{\prime}\right)\right)\right\| \leq \varepsilon
$$

whenever $(x, c)$ and $\left(x, c^{\prime}\right)$ are tagged partitions of $[a, b]$ subordinate to $\delta_{\varepsilon}$.

For an integrable function $f$, a gauge satisfying this property for $\varepsilon$ is said to be $\varepsilon$-adapted (to $f$ ).

Theorem 2.1. Let $f:[a, b] \rightarrow X$ be an integrable function, and for every $\varepsilon>0$, a gauge $\delta_{\varepsilon} \varepsilon$-adapted.

For every family of tagged partitions $\left(\left(x_{\varepsilon}, c_{\varepsilon}\right)\right)_{\varepsilon>0}$ respectively subordinate to $\left(\delta_{\varepsilon}\right)_{\varepsilon>0}$, the function $\varepsilon \rightarrow S_{f}\left(x_{\varepsilon}, c_{\varepsilon}\right)$ has a limit when $\varepsilon$ goes to 0 . This limit does not depend on the sequence chosen. By definition, it is the integral of $f$ on $[a, b]$ and it is denoted by $\int_{a}^{b} f$.

Proof. We first prove the theorem for increasing families of gauges.

Let $\left(\delta_{\varepsilon}\right)_{\varepsilon>0}$ be a family of $\varepsilon$-adapted gauges such that $\delta_{\alpha} \leq \delta_{\beta}$ for $0<$ $\alpha \leq \beta$, and let $\left(\left(x_{\varepsilon}, c_{\varepsilon}\right)\right)_{\varepsilon>0}$ be a family of tagged partitions respectively subordinate to $\left(\delta_{\varepsilon}\right)_{\varepsilon>0}$.

For $0<\alpha \leq \beta$, we can merge the partitions $x_{\alpha}$ and $x_{\beta}$ into a finer one $x_{\alpha, \beta}$. Then, we build $\left(x_{\alpha, \beta}, c_{\alpha, \beta}\right)$ from $\left(x_{\alpha}, c_{\alpha}\right)$ by repeating tags as soon as necessary. The same work can be done from $\left(x_{\beta}, c_{\beta}\right)$ to get $\left(x_{\alpha, \beta}, c_{\beta, \alpha}\right)$.

Thus, $\left(x_{\alpha, \beta}, c_{\alpha, \beta}\right)$ and $\left(x_{\alpha, \beta}, c_{\beta, \alpha}\right)$ are subordinate to $\delta_{\beta}$, and

$$
\left\|S_{f}\left(x_{\alpha}, c_{\alpha}\right)-S_{f}\left(x_{\beta}, c_{\beta}\right)\right\|=\left\|S_{f}\left(x_{\alpha, \beta}, c_{\alpha, \beta}\right)-S_{f}\left(x_{\alpha, \beta}, c_{\beta, \alpha}\right)\right\| \leq \beta .
$$

The family $\left(S_{f}\left(x_{\varepsilon}, c_{\varepsilon}\right)\right)_{\varepsilon>0}$ satisfies the Cauchy property, and it converges.

Now, let $\left(\delta_{\varepsilon}\right)_{\varepsilon>0}$ be a general family of gauges adapted to $f$ and $\left(\left(x_{\varepsilon}, c_{\varepsilon}\right)\right)_{\varepsilon>0}$ a family of subordinate tagged partitions. We have to show the convergence of 
$\left(S_{f}\left(x_{\varepsilon_{n}}, c_{\varepsilon_{n}}\right)\right)_{n \in \mathbb{N}}$ for every decreasing sequence $\left(\varepsilon_{n}\right)_{n \in \mathbb{N}}$ with null limit. Let $\left(\varepsilon_{n}\right)_{n \in \mathbb{N}}$ be such sequence.

We define another family of gauges $\left(\tilde{\delta}_{\varepsilon}\right)_{\varepsilon>0}$ as follows : we set $\tilde{\delta}_{\varepsilon}=\delta_{\varepsilon_{0}}$ for every $\varepsilon \geq \varepsilon_{0}$, and $\tilde{\delta}_{\varepsilon}=\min \left(\delta_{\varepsilon_{0}}, \ldots, \delta_{\varepsilon_{n}}\right)$, whenever $\varepsilon \in\left[\varepsilon_{n}, \varepsilon_{n-1}\left[, n \in \mathbb{N}^{*}\right.\right.$. This family is increasing and obviously adapted to $f$. From the previous result, we conclude that $\left(S_{f}\left(x_{\varepsilon_{n}}, c_{\varepsilon_{n}}\right)\right)_{n \in \mathbb{N}}$ converges. Accordingly, $\varepsilon \mapsto S_{f}\left(x_{\varepsilon}, c_{\varepsilon}\right)$ has a limit when $\varepsilon$ goes to 0 . Finally, let $\left(\delta_{\varepsilon}\right)_{\varepsilon>0}$ and $\left(\delta_{\varepsilon}^{\prime}\right)_{\varepsilon>0}$ two families of gauges adapted to $f$ with respective families of subordinate tagged partitions $\left(\left(x_{\varepsilon}, c_{\varepsilon}\right)\right)_{\varepsilon>0}$ and $\left(\left(x_{\varepsilon}^{\prime}, c_{\varepsilon}^{\prime}\right)\right)_{\varepsilon>0}$. For $\varepsilon>0$, we can build $\delta_{\varepsilon}^{\prime \prime}$ and $\left(x_{\varepsilon}^{\prime \prime}, c_{\varepsilon}^{\prime \prime}\right)$ as follows : $\delta_{\varepsilon}^{\prime \prime}=\delta_{\varepsilon}$ and $\left(x_{\varepsilon}^{\prime \prime}, c_{\varepsilon}^{\prime \prime}\right)=\left(x_{\varepsilon}, c_{\varepsilon}\right)$ whenever $\varepsilon \in \mathbb{Q}_{+}^{*}, \delta_{\varepsilon}^{\prime \prime}=\delta_{\varepsilon}^{\prime}$ and $\left(x_{\varepsilon}^{\prime \prime}, c_{\varepsilon}^{\prime \prime}\right)=\left(x_{\varepsilon}^{\prime}, c_{\varepsilon}^{\prime}\right)$ when $\varepsilon \in \mathbb{R}_{+}^{*} \backslash \mathbb{Q}$.

From above, the functions $\varepsilon \mapsto S_{f}\left(x_{\varepsilon}, c_{\varepsilon}\right), \varepsilon \mapsto S_{f}\left(x_{\varepsilon}^{\prime}, c_{\varepsilon}^{\prime}\right)$ and $\varepsilon \mapsto S_{f}\left(x_{\varepsilon}^{\prime \prime}, c_{\varepsilon}^{\prime \prime}\right)$ have limits which must be the same and the theorem is proved.

\section{Properties of the Integral}

Let $f$ and $g$ be integrable functions on $[a, b]$. The proofs of the following results are identical to the proofs for real-valued functions (see [2] for instance).

\section{Theorem 3.1.}

(i) The functions $f+g$ and $\lambda f$ are integrable for all scalars $\lambda$. Thus, the set of integrable functions has a vector space structure.

(ii) The application $f \mapsto \int_{a}^{b} f$ is a linear form.

(iii) If $f:[a, b] \rightarrow X$ is integrable, the restriction of $f$ to $[r, s] \subset[a, b]$ is integrable (on $[r, s])$. This result allows us to define on $[a, b]$ a function by $F: t \rightarrow \int_{a}^{t} f$.

(iv) Chasles' Relation. Let $a<r<b$ and $f:[a, b] \rightarrow X$ be a function such that restrictions of $f$ to $[a, r]$ and $[r, b]$ are integrable. Then $f$ is integrable on $[a, b]$ and

$$
\int_{a}^{b} f=\int_{a}^{r} f+\int_{r}^{b} f
$$

The following theorem is the main tool to establish more advanced results.

Theorem 3.2. Let $f:[a, b] \rightarrow X$ be an integrable function and $\varepsilon>0$. For every gauge $\delta$-adapted we have

$$
\sum_{i}\left\|\left(x_{i}-x_{i-1}\right) f\left(c_{i}\right)-\int_{x_{i-1}}^{x_{i}} f\right\| \leq \varepsilon
$$


whenever $(x, c)$ is a tagged partition subordinate to $\delta$.

Proof. The proof follows the ideas of the usual Henstock-Saks Lemma.

Let us fix a tagged partition $(x, c)=\left(\left(\left[x_{i-1}, x_{i}\right]\right)_{1 \leq i \leq p},\left(c_{i}\right)_{1 \leq i \leq p}\right)$ subordinate to $\delta$.

Let $\left(\delta_{\varepsilon}\right)_{\varepsilon>0}$ be a family of gauges adapted to $f$. We can assume that $\delta_{\varepsilon} \leq \delta$ for every $\varepsilon>0$ (otherwise, we use $\delta_{\varepsilon}^{\prime}=\min \left(\delta, \delta_{\varepsilon}\right)$ ).

For every integer $i \in\{1, \ldots, p\}$ and $\varepsilon>0$, let $\left(x^{\varepsilon, i}, c^{\varepsilon, i}\right)$ be a tagged partition of $\left[x_{i-1}, x_{i}\right]$ subordinate to the restriction of $\delta_{\varepsilon}$ at this interval. Obviously, we can merge those $p$ tagged partitions to provide a tagged partition $\left(x^{\varepsilon}, c^{\varepsilon}\right)$ of $[a, b]$ subordinate to $\delta_{\varepsilon}$.

From Theorem 2.1, $\lim _{\varepsilon \rightarrow 0} S_{f}\left(x^{\varepsilon, i}, c^{\varepsilon, i}\right)=\int_{x_{i-1}}^{x_{i}} f$.

We can also build a tagged partition $\left(x^{\varepsilon}, d^{\varepsilon}\right)$ by merging the tagged partitions $\left(x^{\varepsilon, i}, d^{\varepsilon, i}\right), 1 \leq i \leq p$, where for every $i$ the sequence of tags $d^{\varepsilon, i}$ is a repetition of the point $c_{i}$. Obviously, $\left(x^{\varepsilon}, d^{\varepsilon}\right)$ is subordinate to $\delta$.

Now, for every $\varepsilon$, the tagged partitions $\left(x^{\varepsilon}, c^{\varepsilon}\right)$ and $\left(x^{\varepsilon}, d^{\varepsilon}\right)$ are subordinate to $\delta$. Collecting packets, we find

$$
\sum_{i=1}^{p} \sum_{j}\left\|\left(x_{j}^{\varepsilon, i}-x_{j-1}^{\varepsilon, i}\right)\left(f\left(c_{i}\right)-f\left(c_{j}^{\varepsilon, i}\right)\right)\right\| \leq \varepsilon,
$$

and

$$
\begin{aligned}
& \sum_{i=1}^{p}\left\|\left(x_{i}-x_{i-1}\right) f\left(c_{i}\right)-\sum_{j}\left(x_{j}^{\varepsilon, i}-x_{j-1}^{\varepsilon, i}\right) f\left(c_{j}^{\varepsilon, i}\right)\right\| \\
& =\sum_{i=1}^{p}\left\|\sum_{j}\left(x_{j}^{\varepsilon, i}-x_{j-1}^{\varepsilon, i}\right)\left(f\left(c_{i}\right)-f\left(c_{j}^{\varepsilon, i}\right)\right)\right\| \leq \varepsilon .
\end{aligned}
$$

We get the result when $\varepsilon$ goes to 0 .

We can prove the continuity of $F: t \mapsto \int_{a}^{t} f$ on $[a, b]$ from Theorem 3.2. In fact, we have a classical stronger result from the generalized Riemann theories.

Theorem 3.3. Let $f:[a, b] \rightarrow X$ be integrable, then the function $F: t \mapsto \int_{a}^{t} f$ is absolutely continuous.

Proof. Let $\varepsilon>0, \delta$ a gauge $\frac{\varepsilon}{2}$-adapted to $f$ and $\left(\left(\left[x_{i-1}, x_{i}\right]\right)_{1 \leq i \leq p},\left(c_{i}\right)_{1 \leq i \leq p}\right)$ a tagged partition subordinate to $\delta$. We set $M=1+\max _{1 \leq i \leq p}\left(\left\|f\left(c_{i}\right)\right\|\right)$ and $\eta=\frac{\varepsilon}{2 M}$. 
Let $\left(\left[r_{k}, s_{k}\right]\right)_{0 \leq k \leq q}$ be a collection of disjoint intervals included into $[a, b]$ such that $\sum_{k=0}^{q}\left(s_{k}-r_{k}\right) \leq \eta$.

Repeating tags $c_{i}$ when necessary, we can include the points $r_{k}$ and $s_{k}$ into the partition. We get a new tagged partition subordinate to $\delta$. From Theorem 3.2 and the triangle inequality, we find

$$
\sum_{k=0}^{q}\left\|F\left(s_{k}\right)-F\left(r_{k}\right)\right\|=\sum_{k=0}^{q}\left\|\int_{r_{k}}^{s_{k}} f\right\| \leq \frac{\varepsilon}{2}+\sum_{k=0}^{q}\left\|\left(s_{k}-r_{k}\right) f\left(c_{k}\right)\right\| \leq \varepsilon,
$$

which is exactly the absolute continuity of $F$.

Theorem 3.4. The Fundamental Theorem of Calculus Let $f:[a, b] \rightarrow$ $X$ be continuous on $[a, b]$. If $f$ is differentiable on $[a, b]$ with $f^{\prime}$ integrable on $[a, b]$, then

$$
\int_{a}^{b} f^{\prime}=f(b)-f(a)
$$

Let us notice that we can find differentiable functions $f$ such that $\left\|f^{\prime}\right\|$ is not integrable. We will see that such functions $f^{\prime}$ cannot be integrable. So, the hypothesis of integrability is necessary and is the weakest one.

Proof. The proof follows the original Henstock's proof [4] with an extra argument.

Let $\varepsilon>0$ and $\delta^{\prime}$ be a gauge $\varepsilon$-adapted to $f^{\prime}$.

For every $t \in[a, b]$, we can find $\delta^{\prime \prime}(t)>0$ such that

$$
\forall u \in[a, b] \cap\left[t-\delta^{\prime \prime}(t), t+\delta^{\prime \prime}(t)\right], \quad\left\|f(u)-f(t)-(u-t) f^{\prime}(t)\right\| \leq \varepsilon|u-t| .
$$

We define $\delta=\min \left(\delta^{\prime}, \delta^{\prime \prime}\right)$. From Borel-Lebesgue Theorem, we can find a subdivision $(x, c)$ subordinate to $\delta$ such that $x_{i-1} \leq c_{i} \leq x_{i}$ for every index [4].

In one hand, from Theorem 3.2 we have $\left\|S_{f^{\prime}}(x, c)-\int_{a}^{b} f^{\prime}\right\| \leq \varepsilon$, on the other hand,

$$
\begin{aligned}
\left\|S_{f^{\prime}}(x, c)-(f(b)-f(a))\right\| \leq & \sum \|\left(x_{i}-x_{i-1}\right) f^{\prime}\left(c_{i}\right)-\left(f\left(x_{i}\right)-f\left(x_{i-1}\right) \|\right. \\
\leq & \sum\left\|\left(x_{i}-c_{i}\right) f^{\prime}\left(c_{i}\right)-\left(f\left(x_{i}\right)-f\left(c_{i}\right)\right)\right\| \\
& +\sum\left\|\left(c_{i}-x_{i-1}\right) f^{\prime}\left(c_{i}\right)-\left(f\left(c_{i}\right)-f\left(x_{i-1}\right)\right)\right\| \\
\leq & \varepsilon \sum\left|x_{i}-c_{i}\right|+\left|c_{i}-x_{i-1}\right|=\varepsilon \sum\left(x_{i}-x_{i-1}\right)= \\
= & \varepsilon(b-a) .
\end{aligned}
$$


So, we have $\left\|\int_{a}^{b} f^{\prime}-(f(b)-f(a))\right\| \leq(1+b-a) \varepsilon$ for every $\varepsilon>0$.

The following result studies the reverse problem and will be useful to establish the measurability of integrable functions.

Theorem 3.5. Let $f:[a, b] \rightarrow X$ be an integrable function and, for all $t \in$ $[a, b]$, let $F(t)=\int_{a}^{t} f$. Then the function $F$ is differentiable almost everywhere on $[a, b]$ and $F^{\prime}(t)=f(t)$ almost everywhere on $[a, b]$.

Proof. The proof of this result is virtually identical to the proof for realvalued functions and the reader is referred to [2] p. 145 for the detail (using left and right derivatives instead of upper and lower left and right derivatives).

\section{When $X$ is a Finite Dimensional Space}

In this section only, the dimension of $X$ is finite.

We want to show that the definitions 1 and 2 provide the McShane theory, itself equivalent to the Lebesgue theory (one more time, we refer to [2] for this result).

Let us recall the McShane integrability definition.

A function $f:[a, b] \rightarrow X$ is McShane-integrable on $[a, b]$ if there exists a vector $\lambda$ with the following properties : for each $\varepsilon>0$ there exists a gauge $\delta$ on $[a, b]$ such that $\left\|S_{f}(x, c)-\lambda\right\| \leq \varepsilon$ whenever $(x, c)$ is a tagged partition of $[a, b]$ subordinate to $\delta$. By definition, $\lambda$ is the McShane integral of $f$ on $[a, b]$.

The following Cauchy criteria is easy to prove.

Theorem 4.1. A function $f:[a, b] \rightarrow \mathbb{R}$ is McShane-integrable on $[a, b]$ if and only if for each $\varepsilon>0$ there exists a gauge $\delta_{\varepsilon}$ such that

$$
\left\|S_{f}(x, c)-S_{f}\left(x^{\prime}, c^{\prime}\right)\right\| \leq \varepsilon .
$$

whenever $(x, c)$ and $\left(x^{\prime}, c^{\prime}\right)$ are tagged partitions of $[a, b]$ subordinate to $\delta$.

Theorem 4.2. For a finite dimension space $X$, a function $f:[a, b] \rightarrow X$ is integrable if and only if it is McShane-integrable. In this case, integrals on $[a, b]$ are the same.

Proof. Let $f$ be an integrable function. For $\varepsilon>0$, let $\delta_{\varepsilon}$ be a gauge on $[a, b] \varepsilon$-adapted (for the definition 1) and $(x, c)$ and $\left(x^{\prime}, c^{\prime}\right)$ two tagged partitions subordinate to $\delta_{\varepsilon}$. Proceeding as in the the proof of Theorem 2.1 (merging of $x$ and $x^{\prime}$ followed by a repetition of tags when needed), we find 
that $\left\|S_{f}(x, c)-S_{f}\left(x^{\prime}, c^{\prime}\right)\right\| \leq \varepsilon$ from the triangle inequality. So, the function $f$ is McShane-integrable.

Conversely, let $f$ be a McShane-integrable function. For $\varepsilon>0$ we can find a gauge $\delta$ satisfying

$$
\sum_{i}\left\|\left(x_{i}-x_{i-1}\right) f\left(c_{i}\right)-\int_{x_{i-1}}^{x_{i}} f\right\| \leq \varepsilon
$$

whenever $(x, c)$ is a tagged partition subordinate to $\delta$ (see [2] for this result). We have to recall that this inequality is only true for $X=\mathbb{R}$ and finite dimension spaces in a wider sense.

Accordingly, for two tagged partitions $(x, c)$ and $\left(x, c^{\prime}\right)$ subordinate to $\delta$, we have

$$
\sum_{i} \|\left(x_{i}-x_{i-1}\right)\left(f\left(c_{i}\right)-f\left(c_{i}^{\prime}\right) \| \leq 2 \varepsilon,\right.
$$

and $f$ is integrable. The integrals are the same from Theorem 2.1.

Let us notice that the above definition can be extended to a general Banach space. Unfortunately, this leads to a theory with non-absolutely integrable functions [3].

\section{$5 \quad$ Integrability of the Norm}

Once again, $X$ is a general Banach space.

From Theorem 4.2 when $X=\mathbb{R}$, we get the following result.

Theorem 5.1. Let $f:[a, b] \rightarrow X$ be an integrable function. Then, the function $\|f\|:[a, b] \rightarrow \mathbb{R}$ is integrable (i.e. is McShane or Lebesgue integrable) and

$$
\left\|\int_{a}^{b} f\right\| \leq \int_{a}^{b}\|f\|
$$

Proof. The triangle inequality applied to the definition 1 for $f$ provides a straightforward proof of the integrability for $\|f\|$.

Moreover, every gauge $\varepsilon$-adapted to $f$ is also $\varepsilon$-adapted to $\|f\|$. Using Theorem 2.1, the final inequality is a simple consequence of $\left\|S_{f}(x, c)\right\| \leq$ $S_{\|f\|}(x, c)$ available for every tagged partition of $[a, b]$.

\section{Measurability of Integrable Functions}

A function $f:[a, b] \rightarrow X$ is called simple if there exists $z_{1}, z_{2}, \ldots, z_{n} \in X$ and $E_{1}, E_{2}, \ldots, E_{p}$ measurable subsets of $[a, b]$ such that $f=\sum_{i=1}^{p} z_{i} \chi_{E_{i}}$, where 
$\chi_{E_{i}}(t)=1$ if $t \in E_{i}$ and $\chi_{E_{i}}(t)=0$ if $t \notin E_{i}$.

A function $f:[a, b] \rightarrow X$ is measurable if it is a pointwise limit of a simple functions sequence.

The usual facts about the stability of measurable functions under sums, scalar multiples and pointwise limits holds.

Lemma 6.1. Let $f:[a, b] \rightarrow X$ be an almost everywhere null function. Then $f$ is integrable with null integral.

Proof. From the hypotheses, $\|f\|$ is null almost everywhere. It is Lebesgueintegrable, and accordingly integrable.

From Theorem 3.2, for each $\varepsilon>0$ there exists a gauge $\delta_{\varepsilon}$ such that

$$
\sum_{i}\left|\left(x_{i}-x_{i-1}\right)\left\|f\left(c_{i}\right)\right\|-\int_{x_{i-1}}^{x_{i}}\|f\|\right|=\sum_{i}\left(x_{i}-x_{i-1}\right)\left\|f\left(c_{i}\right)\right\| \leq \varepsilon
$$

whenever $(x, c)$ is a tagged partition subordinate to $\delta_{\varepsilon}$.

Thus, if $(x, c)$ and $\left(x, c^{\prime}\right)$ are subordinate to $\delta_{\varepsilon}$, we have

$$
\sum_{i}\left\|\left(x_{i}-x_{i-1}\right)\left(f\left(c_{i}\right)-f\left(c_{i}^{\prime}\right)\right)\right\| \leq 2 \varepsilon,
$$

which proves the integrability for $f$. From Theorem 5.1, we have $\int_{a}^{b} f=0$.

Corollary 6.1. Let $f:[a, b] \rightarrow X$ be integrable on $[a, b]$. If $f=g$ almost everywhere on $[a, b]$, then $g$ is integrable on $[a, b]$ and $\int_{a}^{b} f=\int_{a}^{b} g$.

Now, we can prove the main result of this section : the measurability of the integrable functions.

Theorem 6.1. If $f:[a, b] \rightarrow X$ is integrable, then $f$ is also measurable.

Proof. We set $F(t)=\int_{a}^{t} f$ for $t \in[a, b]$. From Theorem 3.5, $f$ is the derivative almost everywhere of $F$. So $f$ is an almost everywhere limit of a sequence of measurable functions and we conclude.

\section{Convergence Theorems}

Let $\left(f_{n}\right)$ be a sequence of integrable functions defined on $[a, b]$ and $X$ valued. The sequence $\left(f_{n}\right)$ is equi-integrable on $[a, b]$ if for each $\varepsilon>0$ there exist a 
gauge $\delta_{\varepsilon}$ on $[a, b]$ such that

$$
\sum_{i}\left\|\left(x_{i}-x_{i-1}\right)\left(f_{n}\left(c_{i}\right)-f_{n}\left(c_{i}^{\prime}\right)\right)\right\| \leq \varepsilon
$$

whenever $(x, c)$ and $\left(x, c^{\prime}\right)$ are tagged subdivisions of $[a, b]$ subordinate to $\delta_{\varepsilon}$.

Just a word about terminology. This notion, well known for generalized Riemann theories, is usually referred as to the uniform integrability. According to it's use for continuous functions, the term "equi-integrability" seems to be better. Just as with uniform continuity, the term "uniform integrability" refers to the integrable function admitting a constant gauge $\delta_{\varepsilon}$ for every $\varepsilon>0$. In consequence, the uniform integrability of a function should be nothing other than its Riemann integrability.

The following theorem is easy to prove and includes the Dominated Convergence Theorem.

Theorem 7.1. Let $\left(f_{n}\right)$ be a sequence of equi-integrable functions on $[a, b]$ and $X$ valued. If $\left(f_{n}\right)$ converges pointwise to $f$ on $[a, b]$ then $f$ is integrable and

$$
\lim _{n \rightarrow+\infty} \int_{a}^{b}\left\|f_{n}-f\right\|=0 \text { and } \lim _{n \rightarrow+\infty} \int_{a}^{b} f_{n}=\int_{a}^{b} f .
$$

Proof. For $\varepsilon>0$, let $\delta_{\varepsilon}$ be a gauge $\varepsilon$-adapted to every functions $f_{n}$. So we have

$$
\forall n \in \mathbb{N}, \quad \sum_{i}\left\|\left(x_{i}-x_{i-1}\right)\left(f_{n}\left(c_{i}\right)-f_{n}\left(c_{i}^{\prime}\right)\right)\right\| \leq \varepsilon
$$

whenever $(x, c)$ and $\left(x, c^{\prime}\right)$ are tagged partitions subordinate to $\delta$.

The integrability of $f$ is a consequence of a simple limit, moreover $\delta$ is also $\varepsilon$-adapted to $f$.

Let $(x, c)$ be a tagged partition subordinate to $\delta$. We know that the sequence $\left(S_{f_{n}}(x, c)\right)_{n \in \mathbb{N}}$ converges toward $S_{f}(x, c)$, and we deduce from Theorem 3.2

$$
\forall n \in \mathbb{N}, \quad\left\|S_{f_{n}}(x, c)-\int_{a}^{b} f_{n}\right\| \leq \varepsilon, \quad \text { and }\left\|S_{f}(x, c)-\int_{a}^{b} f\right\| \leq \varepsilon .
$$

Then $\left\|\int_{a}^{b} f_{n}-\int_{a}^{b} f\right\| \leq 3 \varepsilon$ for large $n$.

The last inequality $\lim _{n \rightarrow+\infty} \int_{a}^{b}\left\|f_{n}-f\right\|=0$ is obtained by applying the above result to the equi-integrable sequence $\left(\left\|f_{n}-f\right\|\right)$. 
Corollary 7.1. Dominated Convergence Theorem Let $\left(f_{n}\right)_{n \in \mathbb{N}}$ be a sequence of integrable functions from $[a, b]$ to $X$ and such that $\left(f_{n}\right)$ converge pointwise almost everywhere to $f$ on $[a, b]$. Suppose there exists an integrable function $g:[a, b] \rightarrow \mathbb{R}_{+}$such that $\left\|f_{n}\right\| \leq g$ almost everywhere and for every integer $n$. Then $f$ is integrable,

$$
\lim _{n \rightarrow+\infty} \int_{a}^{b}\left\|f_{p}-f\right\|=0, \text { and } \lim _{n \rightarrow+\infty} \int_{a}^{b} f_{n}=\int_{a}^{b} f .
$$

Proof. The proof is very close to the proof for real valued functions (using Theorem 3.2 and norms instead of absolute values). We first show that it is enough to prove the result with an everywhere pointwise convergence, then we prove that such a sequence is also equi-integrable. This point is technical and can be obtained from Egorov's Theorem. We refer to [2] for details.

To conclude this section let us establish two properties for the primitives of an equi-integrable sequence.

Theorem 7.2. Let $\left(f_{n}\right)$ be a sequence of equi-integrable functions and suppose $\left(f_{n}\right)$ converges pointwise to $f$ on $[a, b]$. For every $n$, we set $F_{n}(t)=\int_{a}^{t} f_{n}$ and $F(t)=\int_{a}^{t} f$.

(i) The sequence $\left(F_{n}\right)$ converges uniformly to $F$.

(ii) The sequence $\left(F_{n}\right)$ is uniformly absolutely continuous on $[a, b]$.

Proof. Let $\varepsilon>0$ and let $\delta$ be a gauge $\varepsilon$-adapted to the sequence $\left(f_{n}\right)$. We take a tagged partition $(x, c)=\left(\left(\left[x_{i-1}, x_{i}\right]\right)_{1 \leq i \leq q},\left(c_{i}\right)_{1 \leq i \leq q}\right)$ subordinate to $\delta$ and $n_{0}$ such that

$$
\forall n \geq n_{0}, \quad \forall i \in\{1, \ldots, q\}, \quad\left\|f_{n}\left(c_{i}\right)-f\left(c_{i}\right)\right\| \leq \frac{\varepsilon}{b-a} .
$$

Let $M=1+\max \left\{\left\|f_{n}\left(c_{i}\right)\right\|, n \geq n_{0}\right.$ and $\left.1 \leq i \leq q\right\}$.

For the point $(i)$. Let $t \in[a, b]$, and $0 \leq p<q$ such that $x_{p} \leq t<x_{p+1}$. From Theorem 3.2 and the triangle inequality we get

$$
\forall n \in \mathbb{N}, \quad\left\|\sum_{i=1}^{p}\left(x_{i}-x_{i-1}\right) f_{n}\left(c_{i}\right)+\left(t-x_{p}\right) f_{n}\left(c_{p}\right)-F_{n}(t)\right\| \leq \varepsilon .
$$

The gauge $\delta$ is also $\varepsilon$-adapted to $f$, so

$$
\left\|\sum_{i=1}^{p}\left(x_{i}-x_{i-1}\right) f\left(c_{i}\right)+\left(t-x_{p}\right) f\left(c_{p}\right)-F(t)\right\| \leq \varepsilon .
$$


We conclude to $\left\|F_{n}(t)-F(t)\right\| \leq 2 \varepsilon+\frac{\varepsilon}{(b-a)}(b-a)=3 \varepsilon$ as soon as $n \geq n_{0}$.

For $(i i)$, let $\left(\left[r_{i}, s_{i}\right]\right)_{0 \leq i \leq p}$ be a finite collection of disjoint intervals of $[a, b]$. Adding the points $\left(r_{i}\right)$ and $\left(s_{i}\right)$ to $x$ and repeating tags when necessary, we build a new tagged partition $\left(x^{\prime}, c^{\prime}\right)$ subordinate to $\delta$. From Theorem 3.2 we have

$$
\forall n \in \mathbb{N}, \quad \sum_{k=0}^{p}\left\|\sum_{i \text { with } r_{k}<x_{i} \leq s_{k}}\left(x_{i}^{\prime}-x_{i-1}^{\prime}\right) f_{n}\left(c_{i}^{\prime}\right)-\int_{r_{k}}^{s_{k}} f_{n}\right\| \leq \varepsilon .
$$

Then, for $n \geq n_{0}$ we get $\sum_{k=0}^{p}\left\|F_{n}\left(s_{k}\right)-F_{n}\left(r_{k}\right)\right\| \leq 2 \varepsilon$ whenever $\sum_{k=0}^{p}\left(s_{k}-r_{k}\right) \leq$ $\frac{\varepsilon}{M}$.

Now, from the absolute continuity of $F_{0}, \ldots, F_{n_{0}-1}$ and the above inequality, we can find $\eta>0$ such that if $\sum_{k=0}^{p}\left(s_{k}-r_{k}\right) \leq \eta$, then $\sum_{k=0}^{p} \| F_{n}\left(s_{k}\right)-$ $F_{n}\left(r_{k}\right) \| \leq \varepsilon$ for every integer $n$.

\section{Equivalence with the Bochner Theory}

In the following, $\mu$ is the Lebesgue measure on $[a, b]$.

A measurable function $f:[a, b] \rightarrow X$ is called Bochner-integrable if there exists a sequence of simple functions $\left(f_{n}\right)$ such that

$$
\lim _{n \rightarrow+\infty} \int_{a}^{b}\left\|f_{n}-f\right\| d \mu=0 .
$$

Then, the integral of $f$ on $[a, b]$ is defined by $\int_{a}^{b} f=\lim _{n \rightarrow+\infty} \int_{a}^{b} f_{n}$ (which is independent of the defining sequence $\left.\left(f_{n}\right)\right)$.

Theorem 8.1. A function $f:[a, b] \rightarrow X$ is integrable if and only if it is Bochner-integrable. In this case, integrals are the same.

Proof. We first show that every Bochner integrable function is integrable.

Let us recall an important result for integration of Banach-valued functions ([1], corollary 3 p. 42) :

A function $f:[a, b] \rightarrow X$ is measurable if and only if it is the almost everywhere uniform limit of a sequence of countably valued measurable functions. 
From this result, we build a sequence $\left(g_{n}\right)$ of simple functions such that $\int_{a}^{b}\left\|f-g_{n}\right\| \leq 1 / n$ and $\left\|g_{n}\right\| \leq\|f\|+1 / n$ for every $n \geq 1$ (see [1] p. 45).

Moreover, $\lim _{n \rightarrow+\infty} \int_{a}^{b}\left\|f-g_{n}\right\|=0$ implies that we can extract a subsequence $\left(g_{\varphi(n)}\right)$ which converges almost everywhere to $f$.

Now, the functions $g_{\varphi(n)}$ are integrable and dominated by $\|f\|+1$. From the Dominated Convergence Theorem, $f$ is integrable.

The converse implication is a consequence of the following Bochner's Characterization Theorem (see [1]).

Theorem 8.2. A measurable function $f:[a, b] \rightarrow X$ is Bochner-integrable if and only if $\|f\|$ is Lebesgue integrable.

Proof. Let $f$ be an integrable function, from Theorem $6.1 f$ is measurable and $\|f\|$ is Lebesgue-integrable. So, $f$ is Bochner-integrable.

Finally, integrable functions for both theories are pointwise limit of dominated sequences of simple functions and integrals on $[a, b]$ are identical for simple functions. Simple functions are Bochner-integrable and also integrable (because Bochner-integrable!). Using Dominated Convergence Theorems in both theories, we conclude to the equality of the integrals.

Acknowledgement. It is with a great pleasure that I thank A. Warusfel for helpful suggestions and requirements about integration of vector-valued functions.

\section{References}

[1] J. Diestel and J. J. Uhl Jr., Vector measures, Math. Surveys, No. 15, Amer. Math. Soc., Providence, R.I., 1997.

[2] R. A. Gordon, The integrals of Lebesgue, Denjoy, Perron and Henstock, Grad. Studies in Math., vol 4, Amer. Math. Soc. (1994).

[3] R. A. Gordon, The McShane integral of Banach-valued functions, Illinois J. Math. 34(1990), 557-564.

[4] R. Henstock, A Riemann-type integral of Lebesgue power, Canad. J. Math. 20(1968), 79-87.

[5] J. Kurzweil, Generalized ordinary differential equations and continuous dependence on a parameter, Czechoslovak Math. J. 7(82)(1957), 418-446.

[6] E. J. McShane, Unified integration, Academic Press, San Diego, 1983. 